\section{What do we do with a disconnected epidural catheter?}

To the Editor:

Epidural infusions are being used with increasing frequency. If a disconnection between epidural catheter and its luer-lock connector occurs, this may remain undetected for several hours. I conducted a postal survey of anesthesiologists in Canadian teaching hospitals, to ascertain current practice when such a disconnection occurs.

Of the 214 respondents: $2 \%$ would simply reconnect the catheter; $15 \%$ would clean the outside of the catheter and reconnect it; $4 \%$ would cut a portion of the catheter and reconnect it; $44 \%$ would clean the outside of the catheter, cut a portion and reconnect it and $35 \%$ would remove the catheter.

I also asked if there was a departmental policy on action to take when such a disconnection occurs. Twenty-two respondents from nine centres stated that there was, but there was not always consistency in the action taken either between or within these centres. Of the 75 respondents who would remove the catheter 14 stated they were following departmental policy.

When the distal end of an epidural catheter is contaminated with bacteria, they can be cultured from the fluid $87 \mathrm{~cm}$ more proximal, if the fluid meniscus is displaced proximally into the catheter. ${ }^{1}$ A patient of average height, with a thoracic epidural, in an upright position has a vertical height of catheter of approximately $25 \mathrm{~cm}$. Baseline epidural space pressures are $9 \pm$ $5 \mathrm{~cm} \mathrm{H} \mathrm{O}_{2}{ }^{2}$ therefore upon disconnection, fluid will tend to be displaced into the catheter. Bupivacaine, lidocaine, fentanyl and sufentanil used in epidural infusions have been shown to support bacterial growth. ${ }^{3,4}$

Any disconnected catheter should be considered contaminated; increasing time to discovery will increase the risk. Even when $20 \mathrm{~cm}$ of a distal catheter is cut (average length cut is $4.8 \mathrm{~cm}$, range 1 to $20 \mathrm{~cm}$ ), this does not ensure internal sterility no matter how the external of the catheter is cleaned. With fluid displacement into the catheter, the whole of the catheter should be considered contaminated.

Epidural space infection is a rare occurrence. ${ }^{5} \mathrm{We}$ should do all we can to keep it this way. Should we follow the lead of the $35 \%$ of respondents in this survey and remove all epidural catheters that are discovered disconnected in all but exceptional circumstances?

Gareth Parry BM FRCA

Vancouver, British Columbia

\section{References}

1 Langevin PB, Gravenstein N, Langevin SO, Gulig PA. Epidural catheter reconnection. Safe and unsafe practice. Anesthesiology 1996; 85: 883-8.

2 Thomas PS, Gerson JI, Strong G. Analysis of human epidural pressures. Reg Anesth 1992; 17: 212-5.

3 Zaidi S, Healy TE. A comparison of the antibacterial properties of six local analgesic agents. Anaesthesia 1977; 32: 69-70.

4 Feldman JM, Chapin-Robertson K, Turner J. Do agents used for epidural analgesia have antimicrobial properties? Reg Anesth 1994; 19: 43-7.

5 Krauss WE, McCormick PC. Infections of the dural spaces. Neurosurg Clin N Am 1992; 3: 421-33.

\section{Use of the laryngeal mask airway and a modified sequential intubation tech- nique for the management of an unan- ticipated difficult airway in a remote location}

To the Editor:

We report the successful management of a difficult airway with the Laryngeal Mask Airway ${ }^{\circledR}$ (LMA; Nicosia, Cyprus) and a modified sequential intubation technique. We employed this technique, first described for difficult airway management in pediatric patients $^{1-3}$ during an emergency situation outside the operating room.

A lengthy procedure of endovascular radiofrequency ablation, performed in the electrophysiology laboratory in a $160-\mathrm{kg}$ patient with dilated cardiomyopathy and atrial fibrillation, was followed by acute respiratory failure due to pulmonary edema. Manual ventilation was ineffective and attempts at intubation by direct laryngoscopy failed (Cormack- Lehane grade 4). The patient was comatose, hypercapnic and hypoxic (arterial blood gases drawn during the event revealed a $\mathrm{PaO}_{2} 50$ mmHg, a $\mathrm{PaCO}_{2} 85 \mathrm{mmHg}$, a $\mathrm{pH} 7.31$ and $\mathrm{SpO}_{2}$ $78 \%$ ). Prompt insertion of a size 5 LMA allowed recovery of $\mathrm{SpO}_{2}$ to $95 \%$ with $\mathrm{FIO}_{2} 100 \%$.

Subsequently, a modified sequential intubation technique was employed:

l) an endotracheal tube (ET) ID $6.5 \mathrm{~mm}$ was inserted over a fibreoptic bronchoscope (FOB), inserted through the LMA and the trachea was intubated; 2) the FOB was withdrawn after ensuring correct positioning of the ET (tracheal rings and carina visualized) and ventilation resumed $\left(\mathrm{FIO}_{2} 100 \%\right)$ until 